\title{
Rare Persistent Corneal Infection by Phoma sp. - A Case Report
}

\author{
Aspasia Adamopoulou - Dimitris Sakellaris - Spyridon Koronis · \\ Miltos Balidis - Zachos Zachariadis - Paris Tranos - Nikos Kozeis • \\ Zisis Gatzioufas • George Anogeianakis • Dimitrios G. Mikropoulos • \\ Anastasios-Georgios Konstas
}

Received: November 26, 2018 / Published online: January 30, 2019

(c) The Author(s) 2019

\section{ABSTRACT}

We report a case of severe Phoma sp. corneal infection in a middle-aged, otherwise healthy, female patient who was using a soft contact lens. This is the first time that such an infection has been reported in Greece. Our case demonstrates the clinical difficulties and management challenges presented by these recalcitrant

Enhanced digital features To view enhanced digital features for this article go to: https://doi.org/10.6084/ m9.figshare.7588373.

A. Adamopoulou - D. Sakellaris - S. Koronis ·

M. Balidis - Z. Zachariadis - P. Tranos · N. Kozeis .

G. Anogeianakis · D. G. Mikropoulos

Ophthalmica Eye Institute, Vas Olgas 196 and

Ploutonos 27, 54655 Thessaloniki, Greece

Z. Gatzioufas

Augenklinik, Hornhaut, Katarakt und Refractive Chirurgie, Universitaetsspital Basel, Mittlere Strasse 91, 4031 Basel, Switzerland

G. Anogeianakis

Association for Training in Biomedical Technology,

Aristogeitonos 6, 54638 Thessaloniki, Greece

D. G. Mikropoulos

3rd University Department of Ophthalmology,

Aristotle University of Thessaloniki, Thessaloniki, Greece

A.-G. Konstas $(\square)$

1st and 3rd University Departments of Ophthalmology, Aristotle University of Thessaloniki, Thessaloniki, Greece e-mail: konstas@med.auth.gr corneal infections. Management steps included corneal grafting, vitrectomy, and intravitreal antibiotics.

Keywords: Corneal infection; Fungal; Phoma sp.

\section{INTRODUCTION}

Fungal eye infections are rare but can result in keratitis $[1,2]$ and even endophthalmitis. Many different types of fungi can cause eye infections, but the most common infections are caused by species of Fusarium, Aspergillus, Curvularia, and Candida. Trauma is the most frequent predisposing factor, although important contributors include ocular and systemic defects along with the prior application of corticosteroids [3]. Endophytic fungi are ubiquitous organisms found in plants. They usually reside intercellularly or intracellularly without causing any apparent symptoms of infection, while all plants are known to harbor endophytes [4]. Phoma is a genus of endophytic fungus which may also appear on plant foliage. In contrast to most fungal species that can cause eye infections, the involvement of any Phoma sp. in eye injury is previously unreported. In fact, Phoma sp. infection appears to be very rare: among 209 references retrieved in a search in PubMed (lemma: Phoma sp.; last accessed on September 
$19,2019)$, only eight refer (mostly tangentially) to human infection [5-12] (mainly in immunocompromised hosts $[8,12])$, and only one of those may be said to remotely implicate a Phoma sp. in eye infection [5]! Herein, we present a unique case of persistent corneal infection by a Phoma sp. in an immunocompetent adult with no clear origin of infection.

\section{CASE (SUMMARY IN TABLE 1)}

A 47-year-old female who was a long-time soft contact lens user and was meticulous with her lens hygiene presented to her ophthalmologist (March 2014) following a month of constant tearing and progressively worsening blurred vision in the right eye. Anamnesis indicated a preoccupation with gardening and the recent use of commercially available prepackaged plant seeds. The initial diagnosis was bacterial keratitis. She was referred to our center on June 4, 2014 due to a persistent infectious corneal ulcer with a central corneal ulcerative area $(0.75 \mathrm{~mm} \times 1 \mathrm{~mm})$, mild stromal infiltration, and a reduced best-corrected visual acuity of 0.3 . We interrupted topical treatment for $48 \mathrm{~h}$ to obtain culture material (corneal scrapings) and determine the causative agent and her sensitivity to antibiotics. We subsequently initiated treatment (coll. moxifloxacin, coll. azithromycin, washing with Betadine 5\%) according to our standard therapy protocol.

Culture demonstrated the presence of Staphylococcus epidermidis only, while the clinical picture kept worsening. A satellite extension of the infiltration appeared. On June 24, 2014, we biopsied the limits of the infiltration, and local therapy was changed to coll. gentamicin and coll. vancomycin on July 8, 2014 (Fig. 1).

By July 31, 2014, incomplete healing inferiorly with fibrosis was evident. However, central melting ensued, and we sealed the descemetocele with a tectonic graft on August 9, 2014.

Microscopy (under KOH 15\%) of the June 24, 2014 biopsy material revealed abundant narrow and septate fungal hyphae with an acute angle branching pattern. Specimens were inoculated on Sabouraud's dextrose agar with chloramphenicol $(0.05 \%)$, malt extract agar, and
Czapek-Dox agar at $30^{\circ} \mathrm{C}$ and $35^{\circ} \mathrm{C}$, respectively. Following incubation (3-5 days), fungal growth (mold) was present in all cultures, with wrinkled colonies grayish to lightly tanned or light salmon-pink in color that grew at a moderately rapid pace. The mold grew at both $30^{\circ} \mathrm{C}$ and $35^{\circ} \mathrm{C}$ but not at $40^{\circ} \mathrm{C}$. Dark pycnidia with apical openings, abundant small fusiform conidia, and chlamydospores were present. The phenotype was identified as a Phoma sp.

By September 10, 2014, a second lunate extension appeared from the superior border of the tectonic graft. We again biopsied the borders of the new infiltrate, and mycology culture confirmed the previous phenotypic identification, while sequencing of the internal transcribed spacer (ITS1 and ITS2) regions of the isolate's ribosomal DNA presented the highest homology with Phoma foliaceiphila.

As susceptibility testing was not yet complete, we started treatment with ketoconazole $200 \mathrm{mg}$ b.i.d. systemically and topical application of fluconazole hourly. By 15 Sept 2014, hypopyon developed (1.5 $\mathrm{mm}$ height) with paracentral ulceration and melting adjacent to the graft (Fig. 2).

By October 5, 2014, despite antifungal treatment, the whitish infiltrate at the superior border of the graft had enlarged, and there was paracentral melting. We changed treatment to topical amphotericin $0.015 \%$ hourly and to intrastromal injections of amphotericin in order to overcome the limited permeability of the intact epithelium.

On October 15, 2014, we proceeded with a tectonic $7.5 \mathrm{~mm}$ diameter full-thickness keratoplasty, anterior chamber washing, and intrastromal injection of amphotericin $B$ at the graft edges. Additionally, we performed a corneal biopsy and sent specimens to facilitate the identification of the fungus.

Susceptibility results became available on November 7, 2014, which showed in vitro susceptibility to amphotericin B, itraconazole, voriconazole, posaconazole, and micafungin, and resistance to flucytosine and caspofungin. We changed treatment to voriconazole $400 \mathrm{mg}$, twice daily systemically.

The graft remained clear for 2 months with no clinical signs of infection. However, by 
Table 1 Timeline of the case

February 2014

March 2014

June 4, 2014

June 6, 2014

June 24, 2014

July 8, 2014

July 30, 2014

August 9, 2014

September 10, 2014

September 15, 2014

October 5, 2014

October 15, 2014

November 7, 2014

November 21, 2014

December 19, 2014

December 25, 2014 to January 1, 2015

January 2, 2015

March 16, 2015

June 10, 2015

July 1,2015

July 2, 2015

\section{Initial symptoms appear}

Visit to ophthalmologist

Referral to Ophthalmica; persistent infectious corneal ulcer with a central corneal ulcerative area $(0.75 \mathrm{~mm} \times 1 \mathrm{~mm})$, mild stromal infiltration, VA: $3 / 10$

Corneal scrapings obtained (Staphylococcus epidermidis); coll. moxifloxacin, coll. azithromycin, washing with Betadine 5\%

Lunate extension of the infiltration; biopsy of its limits (Phoma sp.)

Coll. gentamicin, coll. vancomycin

Incomplete healing inferiorly with fibrosis

Central melting; sealed descemetocele with tectonic graft

New lunate extension at superior border of the tectonic graft; biopsy of its limits (Phoma sp.); ketoconazole $200 \mathrm{mg}$ b.i.d. systemically and topical application of fluconazole hourly

Hypopyon $(1.5 \mathrm{~mm})$ with paracentral ulceration and melting adjacent to the graft

Enlarged whitish infiltrate at superior border of the graft and paracentral melting; topical amphotericin $0.015 \%$ hourly and intrastromal injections of amphotericin

Tectonic $7.5 \mathrm{~mm}$ diameter full-thickness keratoplasty, anterior chamber washing and intrastromal injection of amphotericin B at the graft edges; corneal biopsy (Phoma foliaceiphila)

Susceptibility results: susceptible to amphotericin B, itraconazole, voriconazole, posaconazole, and micafungin; resistant to flucytosine and caspofungin; voriconazole $400 \mathrm{mg}, \mathrm{s}: 1 \times 2$

Small suture infiltration appears; immediate suture removal and intrastromal injection

Infiltration has invaded the graft and enlarged, despite continuing systemic voriconazole administration

Infiltrate has developed central melting with hypopyon and perforation

Second graft. Reconstruction of anterior chamber, removal of crystalline lens, anterior segment wash-on and core (open sky) vitrectomy, with removal of retroiridic inflammatory membranes and injection of combination of amphotericin-voriconazole into the vitreal cavity; systemic treatment with voriconazole ( $200 \mathrm{mg}$ b.i.d.) and posaconazole started, initially for three months

Clear graft; diminished peripheral neovascularization. Best-corrected VA: 5/10

Discontinued systemic voriconazole

Infiltrate arising from the posterior surface of the iris

Panophthalmitis with hypopyon and posterior vitritis 
Table 1 continued

July 5, 2015

August 2016

January 2017
Emergency PP vitrectomy and silicon oil; restarted systemic voriconazole

Discontinued systemic voriconazole

Infection is under control

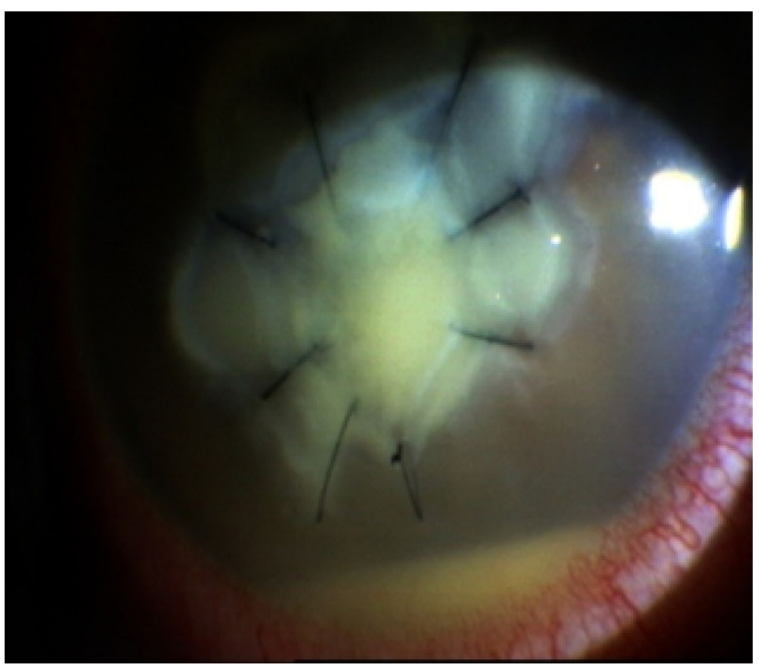

Fig. 1 Right cornea with the initial infiltration and the added lunate infiltration 20 days later. The central corneal ulcer with the stromal infiltration and hypopyon are clearly seen

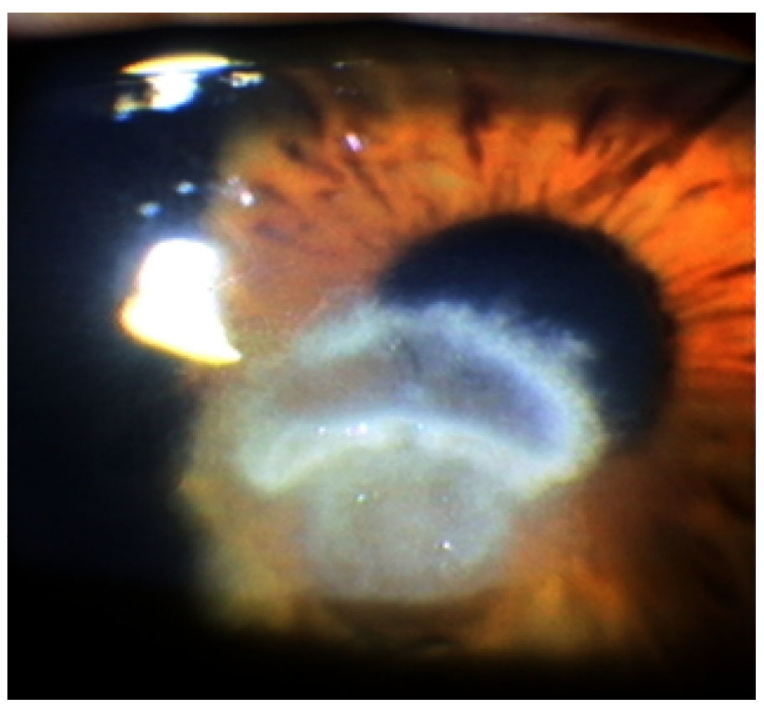

Fig. 2 Appearance of the treated ulcer
November 21, 2014, a small suture infiltration had appeared, which had invaded the graft and enlarged by December 19, 2014 despite systemic voriconazole administration, immediate suture removal, and intrastromal injection.

Between December 25, 2014 and January 1, 2015, the infiltrate developed central melting with hypopyon, and perforation occurred. At this point, a dilemma arose as to whether we should perform enucleation, given the risk of dissemination of the fungal infection to the brain. We decided against it. On January 2, 2015 , we proceeded with a second graft, despite the active, uncontrolled infection. We reconstructed the anterior chamber, removed the crystalline lens, and performed anterior segment wash-on and core (open sky) vitrectomy, with the removal of retroiridic inflammatory membranes and the injection of a combination of amphotericin-voriconazole into the vitreal cavity. Systemic treatment with voriconazole (200 mg b.i.d.) and posaconazole was initiated for an initial period of 3 months, and biweekly liver and kidney function tests were ordered.

By March 15, 2015, the graft remained clear while peripheral neovascularization diminished following two subconjunctival injections of steroid and bevacizumab. Best-corrected VA improved to 0.5 .

On June 10, 2015, we discontinued systemic voriconazole. However, on July 1, 2015, an infiltrate arose from the posterior surface of the iris. By July 2, 2015, panophthalmitis had developed with hypopyon and posterior vitritis, in spite of a clear graft. Consequently, on July 5, 2015, we proceeded with emergency pars plana (PP) vitrectomy and silicon oil. We also restarted systemic voriconazole administration.

As of January 2017, the infection is under control. Systemic voriconazole was 
discontinued 14 months after the last operation (vitrectomy), and silicone oil gradually filled the anterior chamber, damaging the endothelium. This case is reported with the informed consent of the patient.

\section{DISCUSSION}

Based on the literature reported in PubMed, human Phoma ssp. infections are extremely rare, and those reported in the literature involve the lung interstitium [6], the exposed skin of farmers $[7,10]$, or immunocompromised patients $[8,11]$. In addition, in the two cases where the investigation determined (either empirically [7] or through culturing [8]) a Phoma ssp., antibiotic susceptibility pointed to the same basic treatment options: itraconazole, ketoconazole, and amphotericin B $[7,8]$. Fluconazole may sometimes be effective [7], although a Phoma ssp. has been reported to be resistant to both fluconazole as well as 5-flucytosine [8]. It should also be noted that targeted delivery of voriconazole by intrastromal corneal injection is considered a safe and highly effective way to treat deep recalcitrant fungal keratitis [12].

A prominent feature of our case was the apparent low susceptibility of the Phoma sp. to antibiotic/antifungal treatment. However, Phoma ssp. isolated from different medicinal plants has been reported to be a promising source of antimicrobial compounds [13]. This could explain the low susceptibility of the Phoma sp. in our case, as it is reasonable to expect that it must have evolved resistance to its own antibiotic products and related compounds.

We have not been able to determine our patient's infection route. However, the occurrence of major fungi varies with season or with leaf age. The occurrence of Phoma ssp. has been reported to be strongly correlated with season, reaching its maximum-in at least one meticulously investigated case-between the months of February and May [14]. Although this provides only a circumstantial indication, our patient's anamnesis indicated that her infection correlated well with the seasonal peak in Phoma sp. occurrence!

\section{ACKNOWLEDGEMENTS}

Funding. No funding or sponsorship was received for this study or the publication of this article.

Authorship. All named authors meet the International Committee of Medical Journal Editors (ICMJE) criteria for authorship for this article, take responsibility for the integrity of the work as a whole, and have given their approval for this version to be published.

Disclosures. Aspasia Adamopoulou, Dimitris Sakellaris, Spyridon Koronis, Miltos Balidis, Zachos Zachariadis, Paris Tranos, George Anogeianakis and Dimitrios G. Mikropoulos have nothing to disclose. Nikos Kozeis, Zisis Gatzioufas and Anastasios-Georgios Konstas are members of the journal's Editorial Board.

Compliance with Ethics Guidelines. This case is reported with the informed consent of the patient.

Data Availability. Data sharing is not applicable to this article as no datasets were generated or analyzed during the current study.

Open Access. This article is distributed under the terms of the Creative Commons Attribution-NonCommercial 4.0 International License (http://creativecommons.org/licenses/ by-nc/4.0/), which permits any noncommercial use, distribution, and reproduction in any medium, provided you give appropriate credit to the original author(s) and the source, provide a link to the Creative Commons license, and indicate if changes were made.

\section{REFERENCES}

1. Kumar P, Thomas S, Papagiannuli E, Hardman SC, Jenkins D, Prydal J. A case of Phoma fungal keratitis in a contact lens user. JRSM Open. 2015;18:2054270415577760.

2. Rishi K, Font RL. Keratitis caused by an unusual fungus, Phoma species. Cornea. 2003;22:166. 
3. Thomas PA. Fungal infections of the cornea. Eye. 2003;17:852-62.

4. Kaul S, Gupta S, Ahmed M, Dhar MK. Endophytic fungi from medicinal plants: a treasure hunt for bioactive metabolites. Phytochem Rev. 2012;11:487-505.

5. Isa-Isa R, García C, Isa M, Arenas R. Subcutaneous phaeohyphomycosis (mycotic cyst). Clin Dermatol. 2012;30:425-31.

6. Metzger F, Haccuria A, Reboux G, Nolard N, Dalphin J-C, Vuyst PD. Hypersensitivity pneumonitis due to molds in a saxophone player. Chest. 2010;138:724-6.

7. Suh MK. Phaeohyphomycosis in Korea. Nippon Ishinkin Gakkai Zasshi. 2005;46:67-70.

8. Everett J, Busick N, Sielaff T, Wahoff D, Dunn D. A deeply invasive Phoma species infection in a renal transplant recipient. Transpl Proc. 2003;35:1387-9.

9. Tarlo SM, Fradkin A, Tobin RS. Skin testing with extracts of fungal species derived from the homes of allergy clinic patients in Toronto, Canada. Clin Exp Allergy. 1988;18:45-52.
10. Baker JG, Salkin IF, Forgacs P, Haines JH, Kemna ME. First report of subcutaneous phaeohyphomycosis of the foot caused by Phoma minutella. J Clin Microbiol. 1987;25:2395-7.

11. Young NA, Kwon-Chung KJ, Freeman J. Subcutaneous abscess caused by Phoma sp. resembling Pyrenochaeta romeroi: unique fungal infection occurring in immunosuppressed recipient of renal allograft. Am J Clin Pathol. 1973;59:810-6.

12. Kalaiselvi G, Narayana S, Krishnan T, Sengupta S. Intrastromal voriconazole for deep recalcitrant fungal keratitis: a case series. $\mathrm{Br} \mathrm{J}$ Ophthalmol. 2015;99:195-8.

13. Wang L-W, Xu B-G, Wang J-Y, Su Z-Z, Lin F-C, Zhang C-L, et al. Bioactive metabolites from Phoma species, an endophytic fungus from the Chinese medicinal plant Arisaema erubescens. Appl Microbiol Biotechnol. 2011;93:1231-9.

14. Osono T. Endophytic and epiphytic phyllosphere fungi of Camellia japonica: seasonal and leaf agedependent variations. Mycologia. 2008;100(3):387-91. 